\title{
THE POSET OF SKEW FIELDS GENERATED BY A FREE ALGEBRA
}

\author{
JAMES L. FISHER ${ }^{1}$
}

\begin{abstract}
This paper shows that the poset of skew fields generated by a free algebra contains a subposet isomorphic to the lattice of all subsets of an infinite set.
\end{abstract}

Let $R$ be a ring with identity. An $R$-field $(K, \lambda)$ is a skew field $K$ with an identity preserving ring homomorphism $\lambda$ from $R$ into $K$. If $\lambda$ is understood from context, $(K, \lambda)$ will be written as $K$. The $R$-field $(K, \lambda)$ is an epic $R$-field if $K$ is the smallest sub skew field of $K$ containing $\lambda(R)$. Call two epic $R$-fields $\left(K_{1}, \lambda_{1}\right)$ and $\left(K_{2}, \lambda_{2}\right)$ equivalent if there exists an isomorphism $\gamma$ from $K_{1}$ onto $K_{2}$ such that $\gamma \lambda_{1}=\lambda_{2}$. By abuse of notation, epic $R$-field will refer to an equivalence class of epic $R$-fields. For epic $R$-fields $\left(K_{1}, \lambda_{1}\right)$ and $\left(K_{2}, \lambda_{2}\right)$ define $\left(K_{1}, \lambda_{1}\right) \geqq\left(K_{2}, \lambda_{2}\right)$ if there exists a local subring $L$ of $K_{1}$ with $L \supseteq \lambda_{1}(R)$, and a homorphism $\gamma$ from $L$ onto $K_{2}$ such that $\gamma \lambda_{1}=\lambda_{2}$. A straightforward extension of [4, Lemma 1] shows that $\geqq$ is a partial ordering on the set of epic $R$-fields. This partially ordered set will be denoted by $\mathscr{F}_{R}$.

The set of epic $R$-fields $(K, \lambda)$ with $\lambda$ a one-to-one map is a subposet to be denoted by $\mathscr{G}_{R}$. For a commutative ring, $\mathscr{G}_{R}$ either contains one element or is empty depending on whether $R$ is or is not an integral domain. For the ring $R$ of polynomials in noncommuting variables with coefficients in the skew field $k$ (hereafter called a free $k$-algebra), the structure is much richer. Moufang [9] in 1937 proved that $\mathscr{G}_{R}$ is not empty. Neumann [10] in 1949 proved that $\left|\mathscr{G}_{R}\right| \geqq 2$. Amitsur [1] in 1966 proved that $\mathscr{G}_{R}$ has a unique maximal element. Fisher [4] in 1971 proved that $\mathscr{G}_{R}$ contains infinitely many minimal elements. Cohn [2] gave a characterization for general rings in terms of sets of matrices over $R$ called prime matrix ideals. Fisher [5] gave a characterization for general algebras $R$ over infinite fields in terms of right Ore $R$-domains and homomorphisms.

Received by the editors February 23, 1973.

AMS (MOS) subject classifications (1970). Primary 16A06, 16A40; Secondary 16A02, $16 \mathrm{A08}$.

Key words and phrases. Skew field, free algebra, ordered group, epic $R$-field, power series ring, poset.

1 Research supported by the National Research Council of Canada. 
This paper shows the following: Let $F$ be the free group on the set $X$ where the cardinality of $X$ is at least two. Define $F^{\prime \prime}=[[F, F],[F, F]]$. Let $k$ be a skew field and $R$ the free $k$-algebra on the set $X$.

THEOREM 1. $\mathscr{G}_{R}$ contains a subposet corresponding to the set of normal subgroups $H$ of $F$ with $H \subseteq F^{\prime \prime}$ and $F / H$ orderable, where $H_{1} \geqq H_{2}$ if $H_{2} \supseteq H_{1}$ and $H_{2} / H_{1}$ is a convex normal subgroup of $F / H_{1}$ under some ordering of $F / H_{1}$.

THEOREM 2. $\mathscr{G}_{R}$ contains a subposet isomorphic to the lattice of all subsets of an infinite set.

In order to prove these theorems, we will use formal power series ([10] or see [6]). Let $G$ be an ordered group and $k$ a skew field. If $\phi$ is a map from $G$ into $k$, then $\operatorname{supp}(\phi)=\{g \in G: \phi(g) \neq 0\}$. Define $D(G)=$ $\{\phi: \operatorname{supp}(\phi)$ is well ordered in the full ordering of $G\}$. Addition and multiplication in $D(G)$ are given by

$$
(\phi+\psi)(g)=\phi(g)+\psi(g), \quad(\phi \psi)(g)=\sum_{h l=g} \phi(h) \psi(l) .
$$

It is proven in [10] that there is only a finite number of nonzero terms in the summation, and that $D(G)$ is a skew field. Furthermore $O(G)=$ $\{\phi \in D(G): g \geqq e$ for all $g \in \operatorname{supp}(\phi)\}$ is a valuation ring with corresponding valuation $v(\phi)=\min \{\operatorname{supp}(\phi)\}[11, \mathrm{p} .24]$. The invariant primes of the associated valuation ring $O(G)$ correspond exactly to the convex normal subgroups of $G$ [11, p. 14]. Furthermore, if $H$ is a normal convex subgroup of $G$ with corresponding invariant prime $P$ then $O(G) \backslash P$ forms a right Ore system [11, p. 15] and the localization $S(G, H)$ maps naturally onto $D(G / H)$. Define $K(G)$ to be the smallest sub skew field of $D(G)$ containing the group ring $k G$. Let $G_{1}$ and $G_{2}$ be ordered groups which are isomorphic under an isomorphism $\alpha$ as groups (but not necessarily as ordered groups). Let $\lambda_{i}$ be the natural isomorphisms of the group rings $k G_{i}$ into $K\left(G_{i}\right)$, $i=1,2$. Hughes [8] has shown that there exists an isomorphism $\beta$ of $K\left(G_{1}\right)$ onto $K\left(G_{2}\right)$ such that $\beta \lambda_{1}=\lambda_{2} \alpha$. Hence with an orderable group $G$ we associate the (unique up to $k G$-preserving isomorphism) skew field $K(G)$. Thus for a normal subgroup $H$ which is convex under some ordering of $G, S(G, H) \cap K(G)$ is a local subring of $K(G)$ which maps homomorphically onto $K(G / H)$. Hence $K(G) \geqq K(G / H)$.

The proof of Theorem 1 will now be completed with the following lemma.

LEMMA 3. The free semigroup on $X$ is embedded in $F / F^{\prime \prime}$.

Proof. The proof follows easily from the matrix representation of $F / F^{\prime \prime}$ contained in [7]. 
Thus the free $k$-algebra on $X$ is a subalgebra of $k F / H$ where $H$ is a normal subgroup of $F$, and $H \subseteq F^{\prime \prime}$. If in addition $F / H$ is orderable then $K(F / H) \in \mathscr{G}_{R}$. It is clear that for $H_{1}$ and $H_{2}$ distinct normal subgroups of $F$ with $H_{i} \subseteq F^{\prime \prime}, K\left(F / H_{1}\right)$ is not isomorphic to $K\left(F / H_{2}\right)$ as $R$-fields although it is entirely possible they may be isomorphic as skew fields. Hence Theorem 1 is proven.

Proof of Theorem 2. By [7] there exists a representation of $F /\left[F^{\prime \prime}, F\right]$ onto a group $M$ of matrices. The kernel of the representation is a subgroup properly contained in $F^{\prime \prime} /\left[F^{\prime \prime}, F\right] . M^{\prime \prime}$ is central and free abelian on an infinite number of generators. Hence each subset $S$ of the free generator set $Y$ of $M^{\prime \prime}$ generates a central subgroup $H(S)$ of $M$. The group $M / H(S)$ is orderable since $F / F^{\prime \prime} \cong M \mid M^{\prime \prime}$ is orderable and $M^{\prime \prime} \mid H(S)$ is central free abelian, isomorphic to $H(Y \backslash S)$. If $S_{1} \subset S_{2}$ then $H\left(S_{1}\right) \subset H\left(S_{2}\right)$ and $H\left(S_{2}\right) / H\left(S_{1}\right)$ is a convex normal subgroup under an appropriate ordering of $M / H\left(S_{1}\right)$. Thus, by Theorem $1, K\left(M / H\left(S_{1}\right)\right) \geqq K\left(M / H\left(S_{2}\right)\right)$ and $\mathscr{G}_{R}$ contains a poset isomorphic to the lattice of subsets of $Y$.

COROLlaRY. $\mathscr{G}_{R}$ contains infinite ascending and descending chains.

ACKNOWLedgement. I would like to thank Akbar Rhemtulla and Stewart Steinberg for their helpful comments.

\section{REFERENCES}

1. S. A. Amitsur, Rational identities and applications to algebra and geometry, J. Algebra 3 (1966), 304-359. MR 33 \#139.

2. P. M. Cohn, Free rings and their relations, Academic Press, London and New York, 1971.

3. - Rings of fractions, University of Alberta Lecture Notes, 1972.

4. J. L. Fisher, Embedding free algebras in skew fields, Proc. Amer. Math. Soc. 30 (1971), 453-458. MR 43 \#7465.

5. - The category of epic R-fields, J. Algebra (to appear).

6. L. Fuchs, Partially ordered algebraic systems, Pergamon Press, New York; Addison-Wesley, Reading, Mass., 1963. MR 30 \#2090.

7. C. K. Gupta, A faithful matrix representation for certain centre-by-metabelian groups, J. Austral. Math. Soc. 10 (1969), 451-464. MR 41 \#5513.

8. I. Hughes, Division rings of fractions for group rings, Comm. Pure Appl. Math. 23 (1970), 181-188. MR 41 \#8533.

9. R. Moufang, Einige Untersuchungen über geordnete Schiefkörper, J. Reine Angew. Math. 196 (1937), 203-223.

10. B. H. Neumann, On ordered division rings, Trans. Amer. Math. Soc. 66 (1949), 202-252. MR 11, 311.

11. O. F. G. Schilling, The theory of valuations, Math. Surveys, no. 4, Amer. Math. Soc., Providence, R.I., 1950. MR 13, 315.

Department of Mathematics, University of Alberta, Edmonton, Alberta, CANADA 